\title{
Proliferative Verrucous Leukoplakia of the Gingiva, Report of two Cases with Malignant Transformation
}

\author{
Nadereh Ghanee DMD, Selene Saraf, Mary Nathanson Kilo MD and Kristina Waggoner \\ Pacific Northwest Kaiser Dental, USA
}

${ }^{\star}$ Corresponding author: Selene Saraf, University of Washington undergraduate student and Northwest Kaiser volunteer; Tel: 2069548148; E-mail: selenesaraf@gmail.com

Received Date: October 05, 2017, Accepted Date: November 03, 2017, Published Date: November 06, 2017

Citation: Nadereh Ghanee DMD, et al. (2017) Proliferative Verrucous Leukoplakia of the Gingiva, Report of two Cases with Malignant Transformation. J Clin Anat Pathol 3: 1-6.

\begin{abstract}
Gingival Squamous Cell Carcinoma (SCC) may present with varied clinical and histological appearances. Proliferative verrucous Leukoplakia (PVL) is a high risk non-homogenous leukoplakia. PVL has a high probability of recurrence and has shown a high rate of malignant transformation to either squamous cell carcinoma or verrucous carcinoma. This paper discusses two cases of gingival PVL with malignant transformation. Both patients were female, ages 62 and 70 who were seen at the oral pathology clinic in Kaiser. The initial biopsy results on both cases were suggestive of PVL. Close observation and repeated follow up visits showed recurrent lesions and subsequently early detection of SCC.

Keywords: Gingival Squamous Cell Carcinoma; Proliferative verrucous Leukoplakia
\end{abstract}

\section{Introduction}

Proliferative Verrucous Leukoplakia (PVL) is an aggressive form of leukoplakia with a high recurrence rate and potential for malignant transformation. This type of condition needs to be followed up closely with early and aggressive treatment. The gingiva is frequently involved, but other sites may be affected as well. Unlike leukoplakic lesions, PVL exhibits persistent growth with verrucous appearance. Malignant transformation of PVL usually happens in two stages. The first stage of PVL could be indistinguishable from verrucous carcinoma. The second stage is the development of dysplasia and transformation into squamous cell carcinoma.

\section{Case 1}

A 62 year old female patient was seen in a Pacific Northwest Kaiser Dental facility for a regular dental checkup and cleaning in June of 2008. The patient's medical history included impaired fasting glucose and essential hypertension. She was never a smoker. The attached gingiva, facial of tooth \#8 and \#9 showed diffuse erythema and the patient was asymptomatic. The clinical diagnosis was plasma cell gingivitis and she was prescribed a topical steroid. The patient was re-checked monthly and was showing slight improvement during this time. In December of 2008, a biopsy was performed since the erythema was back.

(c)2017 The Authors. Published by the JScholar under the terms of the Creative Commons Attribution License http://creativecommons.org/licenses/ by $/ 3.0 /$, which permits unrestricted use, provided the original author and source are credited.
The result was, "squamous hyperplasia with lympho-plasmacytic inflammation, consistent with plasma cell gingivitis." Close observation and follow-up was recommended by the pathology team. The patient was checked periodically, showing complete healing of the biopsy site until June of 2013. She came back with leukoplakic lesions with a non-homogenous surface growing on the attached gingiva of teeth \#8 to \#10. A biopsy was performed and the result was, "atypical papillary hyperplasia," and close follow-up was recommended. During the follow-up appointment in December of 2013, it was found that the patient developed prominent verrucous lesions on the buccal and lingual attached gingiva of teeth $\# 8$ to \#10. A complete removal of the lesions were done and the pathology result was, "atypical squamous proliferation, cannot exclude squamous cell carcinoma." The case was sent to the Oregon Health and Sciences University (OHSU) for further consult and their result were features suggestive of a well-differentiated squamous cell carcinoma, consistent with PVL. In May of 2014, the otolaryngology team of Kaiser performed bone resection along with extracting the involved teeth due to regrowth of the lesions. The pathology result was well-differentiated squamous cell carcinoma. The P16 test was negative for HPV. Nuclear medicine studies showed no involvement of adjacent areas. The patient has been free of disease until now and getting regular dental check-ups at Kaiser Dental (Figure1 to Figure 5). 
Figure 1: Case 1) Gingival erythema on facial gingiva of teeth \#8, \#9, biopsy result was "plasma cell gingivitis." Image taken in December, 2008.

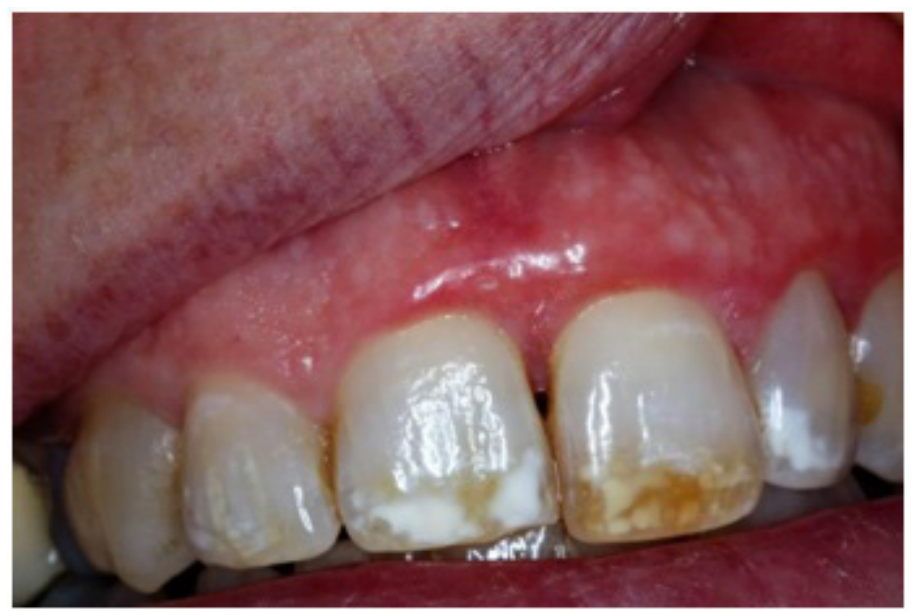

Figure 2: Case 1) Proliferative verrucous leukoplakia, the biopsy result was, "atypical papillary hyperplasia.” Image taken in June, 2013.
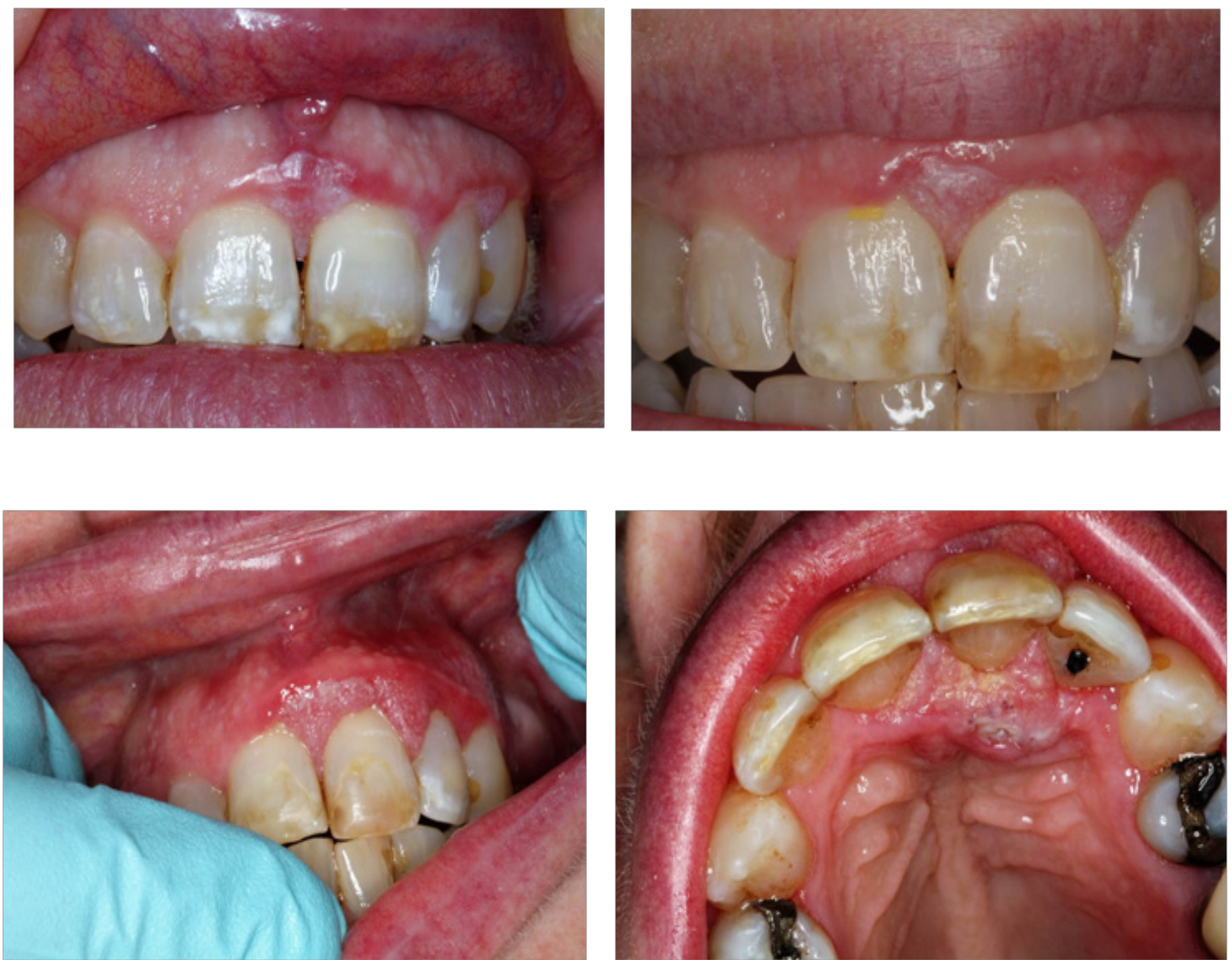

Figure 3: Case 1) Recurrent lesion, pathology result was “atypical squamous proliferation, cannot exclude squamous cell carcinoma." Image taken in December, 2013. 
Figure 4: Case 1) Post biopsy photos two weeks later. Image taken in January, 2014
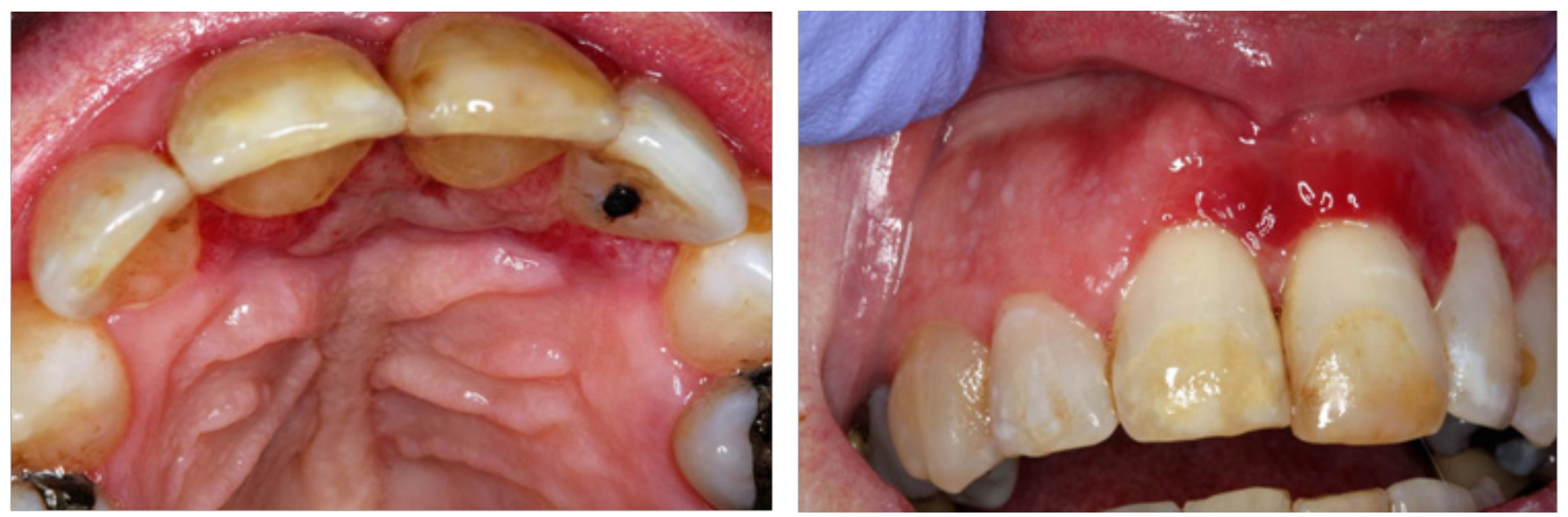

Figure 5: Case 1) Post cancer treatment, including extraction of the involved teeth and bone resection. Image taken in 2016

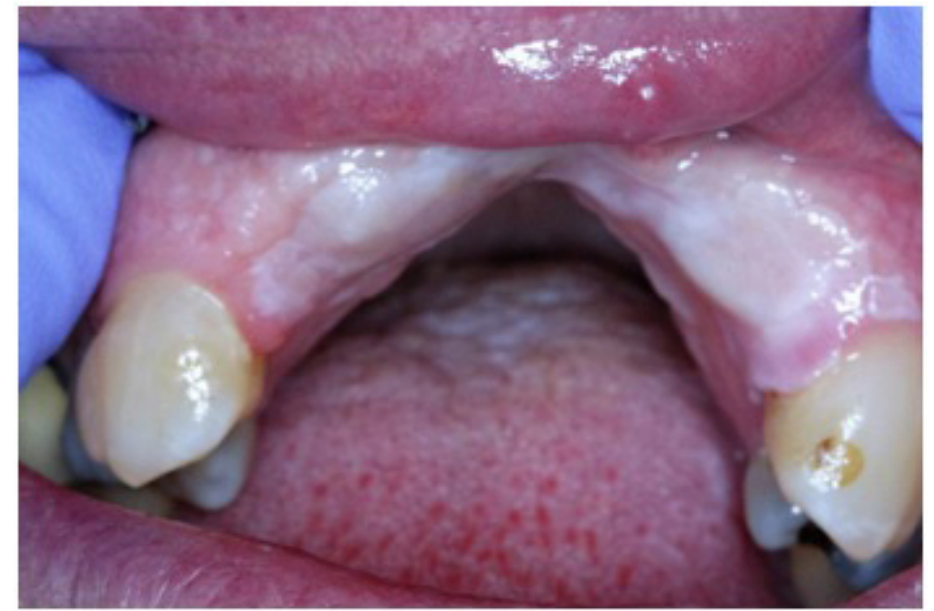

\section{Case 2}

A 70 year old female patient saw a Kaiser periodontist for a bone graft procedure around tooth \#27 in March of 2013. Her medical history included mild arthritis and hypertension. She was not a smoker. The patient's chart revealed that this area had been monitored from 2001 to 2013 due to constant tissue changes. She had repeated biopsies which always resulted in lichenoid inflammation or lichen planus. However, in March of 2013 the clinical appearance of the lesion did not look like lichenoid inflammation. An incisional biopsy was done and the result was, "atypical papillary epithelial hyperplasia with dysplasia and candidiasis." Two weeks after, tooth \#27 was removed along with complete removal of the tissue around it. This excisional biopsy revealed the same result which was confirmed by OHSU's oral pathology team. However, OHSU noted that this process could be PVL and needed close observation and follow-up.
By May of 2013 the site had showed complete healing. However, in July of 2013 mobility of \#26 on the site was noticed. The gingival sulcus and periodontal pocket showed an unusual white keratin-like material as well. A third biopsy was done. The result was, "moderate to severe dysplasia." The patient was then referred to the Kaiser otolaryngology team where bone resection and extraction of the involved teeth were done. The pathology result was "well-differentiated squamous cell carcinoma." The P16 test was negative for HPV. There was no lymph node involvement or invasion of cancer to the adjacent areas. The patient has been free of disease since the last procedure (Figure 6 to Figure 10). 
Figure 6: Case 2) Erythema and inflammation, biopsy result was “consistent with lichen planus." Image taken in 2004
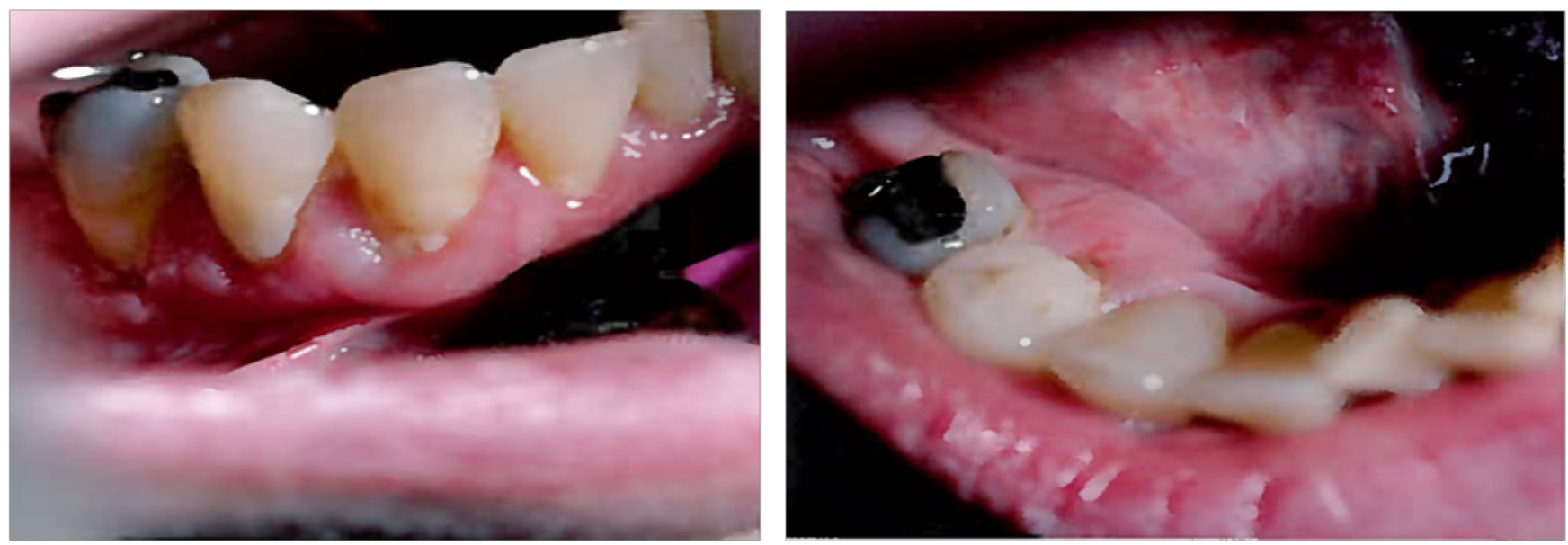

Figure 7: Case 2) Recurrent lesion on distal of \#27, the biopsy result was “consistent with lichen planus.” Image taken in 2005

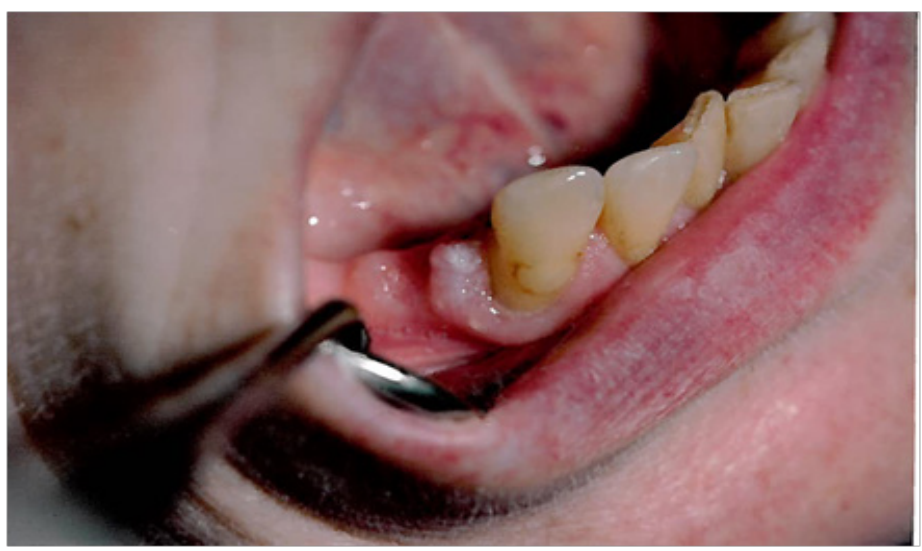

Figure 8: Case 2) Recurrent lesion on distal of \#27, the biopsy result was “epithelia hyperplasia and lichenoid gingivitis." Image taken in 2011

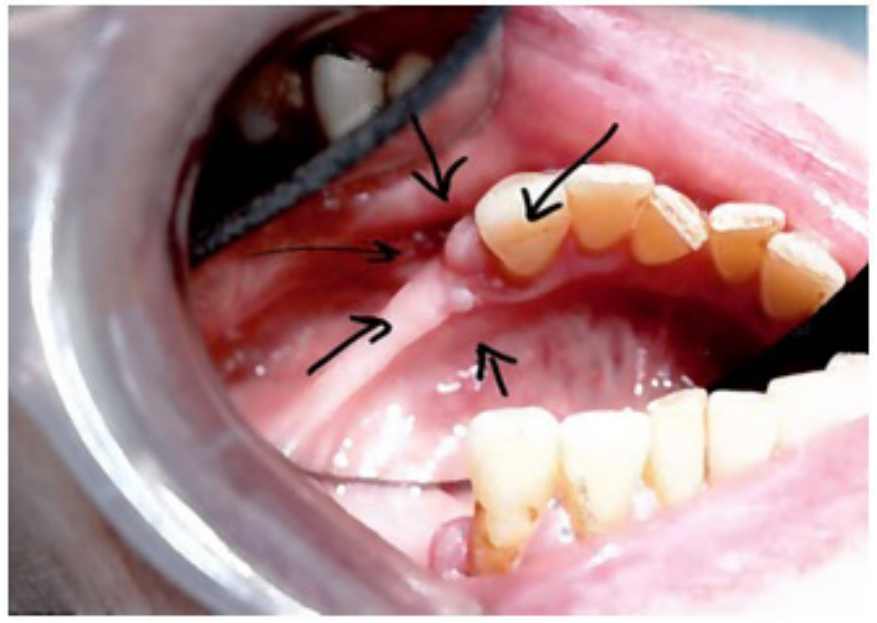


Figure 9: Case 2) Proliferative Verrucous leukoplakia PVL on distal, buccal and lingual of \#27, Image taken in 2013
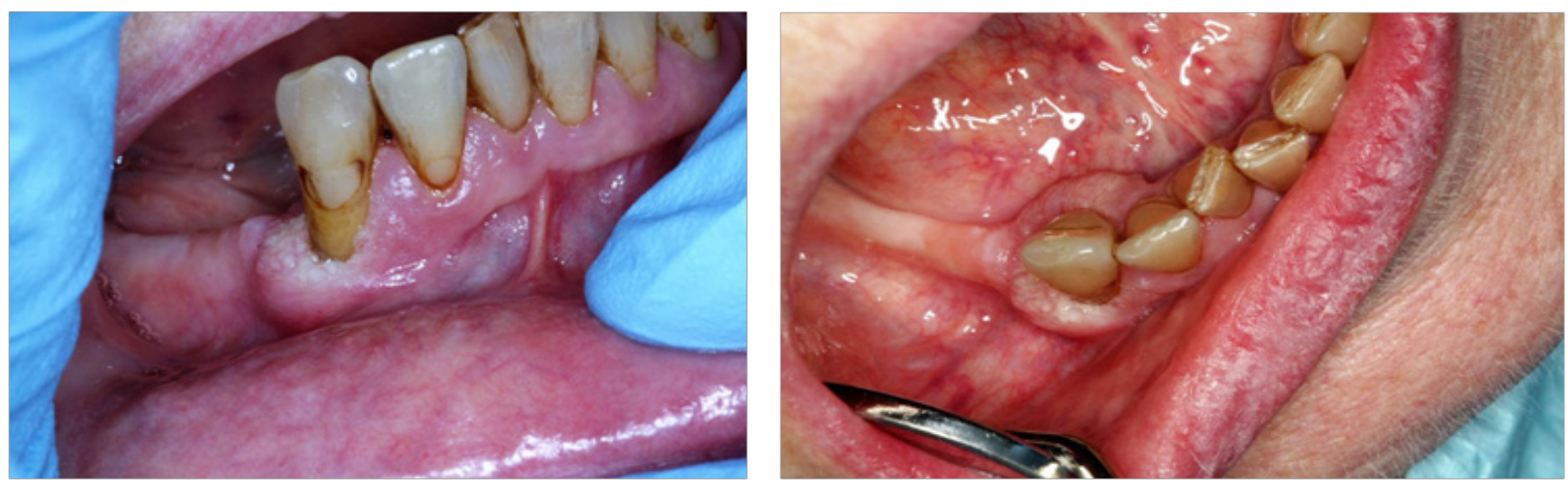

Figure 10: Case 2) Complete removal of the lesion along with extraction of \#27 was done, the area healed in two months
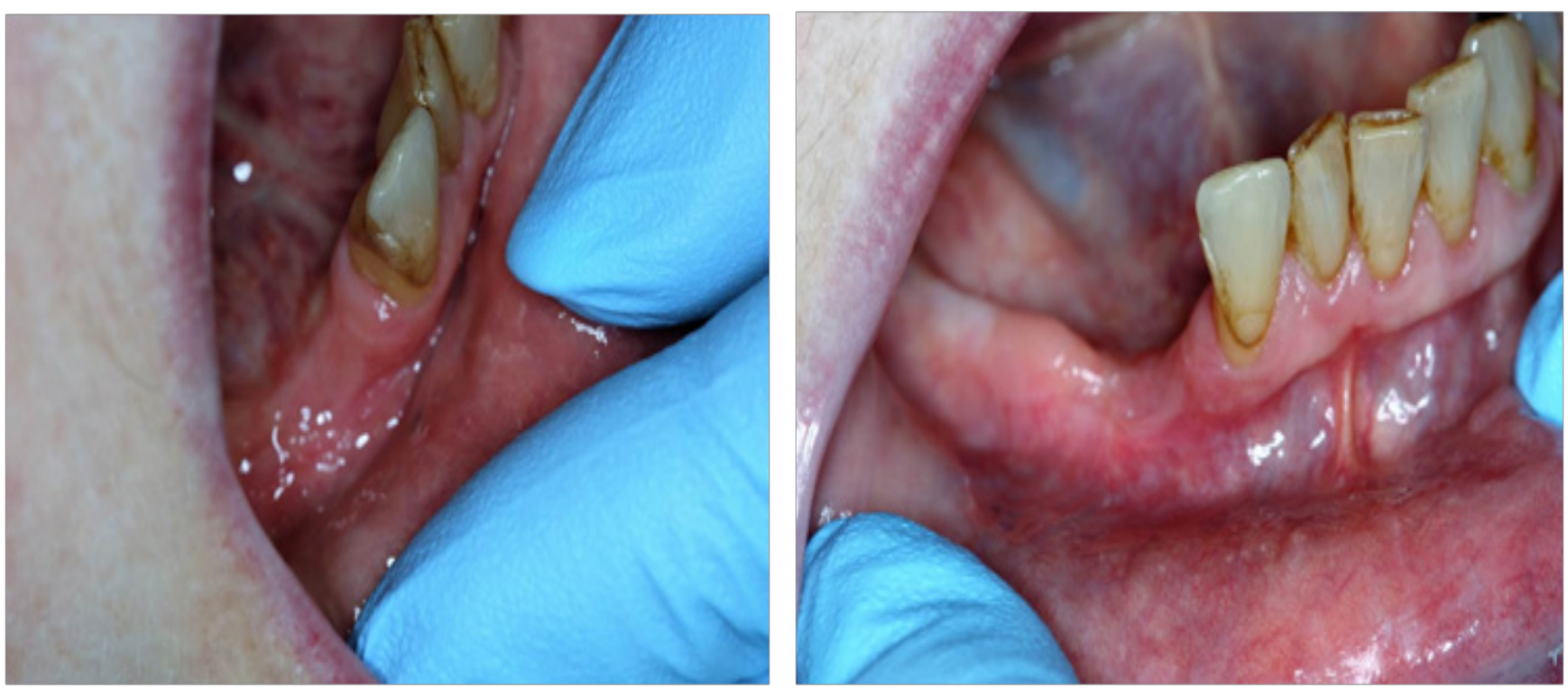

\section{Discussion}

Both cases were female patients, non-smokers and the gingiva was the site of involvement. Bagan et al. evaluated 55 cases of PVL and monitored their malignant transformation. The PVL patients who developed oral cancers were more commonly female and non-smokers. Those patients who developed more than one squamous cell carcinoma were most likely to develop lesions of the gingiva $[1,2]$. PVL was first defined as a new and different form of leukoplakia by Hansen et al. in 1985 [3-6]. It has been described as an aggressive leukoplakia with rapid malignant transformation. Another aspect that is worth noting is that PVL has strong female predilection (1:4 male-to-female ratios) and minimal association with tobacco use. Bagan et al showed the average of the PVL patients to be $70.97+/-12.73$ years [7]. Of these PVL patients, $80 \%$ were women and only $23.3 \%$ were cigarette smokers. Studies have shown recurrences of PVL after treatment in $86.7 \%$ of cases. Recurrences of new lesions during follow-up have been found in $83.3 \%$ and oral cancer eventually in $63.3 \%$, with high incidence on the gingiva [8].
Hansen et al. stated that only $31 \%$ of PVL cases use tobacco products [9]. Alcohol consumption is another possible factor that has not been widely investigated in patients affected by PVL [10]. There is also no correlation between immunodeficiency and PVL. The role of Human Papilloma Virus (HPV) in the origin of PVL has been controversial as well. Some studies show HPV as a co-factor which plays the role in some of the PVL lesions [11]. Fettig et al. could not detect HPV using PCR in 10 patients with recurrent PVL [12]. Hansen et al. followed patients with PVL for 20 years, 13 died of their disease, 14 were alive with PVL, and 3 were alive without PVL at last contact [13]. Like other forms of leukoplakia, PVL may become dysplastic or even malignant with no change in its clinical appearance. It can also progress and go through a stage of indistinguishable from verrucous carcinoma. On the other hand, other forms of leukoplakia can demonstrate gradual scattered patches of redness, making a red-white appearance also called, "erythroleukoplakia," or "speckled leukoplakia." These two cases initially showed erythema and subsequently changed to features of PVL. 
Majority of cases showing diffuse gingival erythema and inflammation can resolve when causative factors are eliminated. Those erythematous gingival conditions include plasma cell gingivitis, allergic contact stomatitis and gingivitis due to mouth breathing and lichenoid reactions. Some autoimmune conditions such as lichen planus can present with erythema, mixed with white striations. Erythematous conditions of the gingiva should be taken seriously with close observation and follow up checks. As shown here, there is potential of transformation to PVL and subsequently to squamous cell carcinoma. Red lesions occurring in the oral cavity if not inflammatory, autoimmune, or traumatic in origin, have potential to be dysplastic with future malignant transformation. Some erythroplakic lesions can microscopically demonstrate dysplasia, carcinoma in situ or squamous carcinoma.

\section{Conclusion}

Close observation and follow up check of red lesions of the gingiva is crucial. Any erythematous gingival lesion or erythema mixed with verrucous leukoplakia necessitates high clinical suspicion. Such cases can microscopically show carcinoma in situ or even squamous cell carcinoma. As seen here, some diffuse erythema of the gingiva, or lichenoid features can transform to PVL and subsequently to squamous cell carcinoma. PVL often has aggressive and lethal behavior. Clinicians should familiarize themselves with the clinical presentation of PVL, since management of PVL is different from traditional leukoplakia.

\section{References}

1) Bagan JV, Jiménez-Soriano Y, Diaz-Fernandez JM, Murillo-Cortés J, Sanchis-Bielsa JM, et al. (2011) Malignant transformation of proliferative verrucous leukoplakia to oral squamous cell carcinoma: a series of 55 cases. Oral Oncol 47: 732-735.

2)Bagan J, Scully C, Jimenez Y, Martorell M (2010) Proliferative verrucous leukoplakia: a concise update Oral Dis 16: 328-332.

3) Hansen LS, Olson JA, Silverman S Jr (1985) Proliferative verrucous leukoplakia: A long-term study of thirty patients. Oral Surg Oral Med Oral Pathol 60: 285-298.

4) Abadie WM, Partington EJ, Fowler CB, Schmalbach CE (2015) Optimal management of proliferative verrucous leukoplakia: a systematic review of the literature. Otolaryngol Head Neck Surg 153: 504-511.

5) Shopper TP, Brannon RB, Stalker WH (2004) Proliferative verrucous leukoplakia: an aggressive form of oral leukoplakia. J Dent Hyg 78: 7.

6) Parashar Pallavi (2016) Insights Into proliferative verrucous leukoplakia. Oral Pathol.

7) Bagan JV, Jiménez-Soriano Y, Diaz-Fernandez JM, Murillo-Cortés J, Sanchis-Bielsa JM, et al. (2011) Malignant transformation of proliferative verrucous leukoplakia to oral squamous cell carcinoma: a series of 55 cases. Oral Oncol 47: 732-735.

8) Louis S Hansen DDS, James A OlsonDDS, SolSilverman Jr DDS (1985) Proliferative verrucous leukoplakia: A long-term study of thirty patients. Oral Surg Oral Med Oral Pathol 60: 285-298.

9) Ibid. Hansen p.1

10) Ibid. Hansen. p. 3

11) Murrah VA, Batsakis JG (1994) Proliferative verrucous leukoplakia and verrucous hyperplasia. Ann Otol Rhinol Laryngol 103: 660-663.

12) Fettig A, Pogrel MA, Silverman S Jr, Bramanti TE, Da Costa M, et al. (2000) Proliferative verrucous leukoplakia of the gingiva. Oral Surg Oral Med Oral Pathol Oral Radiol Endod 90: 723-730.

13) Hansen LS, Olson JA, Silverman S Jr (1985) Proliferative verrucous leukoplakia: A long-term study of thirty patients. Oral Surg Oral Med Oral Pathol 60: 285-298. 